\title{
Korteweg-de Vries solitons on electrified liquid jets
}

\author{
Qiming Wang \\ Department of Mathematics and Statistics, York University, Ontario M3J 1P3, Canada \\ Demetrios T. Papageorgiou \\ Department of Mathematics, Imperial College London, South Kensington Campus, London SW7 2AZ, United Kingdom \\ Jean-Marc Vanden-Broeck \\ Department of Mathematics, University College London, Gower Street, London WC1E 6BT, United Kingdom
}

(Received 15 March 2015; published 25 June 2015)

\begin{abstract}
The propagation of axisymmetric waves on the surface of a liquid jet under the action of a radial electric field is considered. The jet is assumed to be inviscid and perfectly conducting, and a field is set up by placing the jet concentrically inside a perfectly cylindrical tube whose wall is maintained at a constant potential. A nontrivial interaction arises between the hydrodynamics and the electric field in the annulus, resulting in the formation of electrocapillary waves. The main objective of the present study is to describe nonlinear aspects of such axisymmetric waves in the weakly nonlinear regime, which is valid for long waves relative to the undisturbed jet radius. This is found to be possible if two conditions hold: the outer electrode radius is not too small, and the applied electric field is sufficiently strong. Under these conditions long waves are shown to be dispersive and a weakly nonlinear theory can be developed to describe the evolution of the disturbances. The canonical system that arises is the Kortweg-de Vries equation with coefficients that vary as the electric field and the electrode radius are varied. Interestingly, the coefficient of the highest-order third derivative term does not change sign and remains strictly positive, whereas the coefficient $\alpha$ of the nonlinear term can change sign for certain values of the parameters. This finding implies that solitary electrocapillary waves are possible; there are waves of elevation for $\alpha>0$ and of depression for $\alpha<0$. Regions in parameter space are identified where such waves are found.
\end{abstract}

DOI: 10.1103/PhysRevE.91.063012 PACS number(s): 47.15.km, 47.65.-d, 47.35.Fg, 47.35.Pq

\section{INTRODUCTION}

Cylindrical liquid jets that support surface tension are susceptible to long-wave instabilities: any linear perturbation with wavelength longer than the jet circumference is unstable and eventually leads to the breakup of the jet into drops-see Plateau [1] and Rayleigh [2]. Axisymmetric perturbations are found to be the most dangerous ones in the absence of rotation or other external effects. The instability is present for both lowand high-viscosity fluids due to its physical origin - a perfectly cylindrical liquid thread can minimize its surface energy by disintegrating into spherical droplets of equivalent volume. The stabilization of such Rayleigh or capillary instabilities has been the subject of numerous studies that invoke different physical mechanisms to influence the spectrum. Of particular note are mechanisms involving electric and magnetic fields.

Electric DC fields acting along the axis of the jet have been observed to stabilize the Rayleigh instability and enable the formation and persistence of longer liquid bridges; for example, see the experiments and theory of Raco [3], Ramos et al. [4], and Burcham and Saville [5]. These observations were confirmed by numerical solutions of the full equations by Volkov et al. [6]. In the presence of radial electric fields (as in the present study), the jet or liquid cylinder can be stabilized or destabilized depending on the strength of the field and the separation distance between the liquid and the outer cylindrical electrode. To fix things we consider a perfectly conducting liquid jet inside a concentric perfectly cylindrical electrode so that the field acts in the annulus alone. This problem has a long history starting with linear studies by; for example, see Basset [7], Schneider et al. [8], Neukermans [9], and Artana et al. [10,11], who show that the electric field increases the critical Weber number (analogously the effective jet velocity) below which absolute instability is supportedthis may be useful in experimental studies since nonelectrified jets support absolute instabilities at such low speeds that the experiments become very delicate-see Chauhan et al. [12]. The manifestation of the instability into the nonlinear regime and eventual jet pinching has been considered by Setiawan and Heister [13] using boundary-element time-dependent computations. In this paper we also study nonlinear aspects of the problem and in particular we consider nonlinear waves valid in an electric-field-induced long-wave stability window.

Our work is related to the study of Grandison et al. [14], who computed traveling waves of arbitrary amplitude and sufficiently short wavelengths so that they are linearly stable due to capillarity. They considered both perfectly conducting liquid jets, but also perfect dielectric (insulating) ones. In the latter case, a rod electrode needs to be present along the axis of the configuration surrounded by a liquid annulus, which is in turn surrounded by a second annular region adjacent to the outer cylindrical electrode (such flow geometries have been studied in different nonelectrified setups to evaluate the effect of surfactants on thread-annular flows; see, for example, Bassom et al. [15] and references therein). The computations in Ref. [14] are electrified extensions of nonlinear cylindrical traveling waves calculated by Vanden-Broeck et al. [16].

The problem of waves on the surface of liquid jets made of ferrofluids has also received attention over the years. A magnetic field in the azimuthal direction is generated by 
passing a current through a thin wire placed on the axis of the ferrofluid. It has been shown that a sufficiently strong induced magnetic field can stabilize capillary instabilities and ultimately produce nonlinear traveling waves. These are governed by a Kortweg-de Vries (KdV) equation for weakly nonlinear axisymmetric deformations; see Bashtovoi et al. [17] and Rannacher and Engel [18]. Recent experiments by Bourdin et al. [19] have confirmed the existence of axisymmetric depression and elevation solitons that follow KdV dynamics. In a recent theoretical study by Blyth and Parau [20], solitary waves of arbitrary amplitude were computed numerically and the elevation and depression weakly nonlinear solitons found in Ref. [18] were calculated, along with new branches of solitary waves. Large amplitude waves can develop to form toroidal trapped bubbles, as seen by Grandison et al. [14] in a different physical and mathematical setup. The present study (as well as that in Ref. [14]) is more complicated mathematically than those for ferrofluids. The reason for this is that the ferrofluid equations simplify significantly in axisymmetric geometries and the mathematical problem is modified by adding a term to the Bernoulli equation that is inversely proportional to the local jet radius. In the present case the problem is also axisymmetric but electric fields act in the annular region and need to be solved together with the hydrodynamic problem to determine the interfacial position. Nonetheless, results that are analogous to those for ferrofluids emerge, namely, weakly nonlinear $\mathrm{KdV}$-type dynamics along with depression and elevation solitons being supported.

The rest of the paper is organized as follows. Section II formulates the mathematical model and nonlinear boundary conditions and also presents the linear dispersion relation for arbitrary wave numbers. Section III constructs a long-wave weakly nonlinear theory for the coupled electrohydrodyanmic problem that leads to a Kortweg-de Vries-type equation that can support depression or elevation waves depending on the relative values of the electric-field strength and the electrode radius; a phase diagram is calculated that separates depression from elevation waves in the electric field-electrode radius space. Section IV contains some concluding remarks and possible future work.

\section{PROBLEM FORMULATION}

Consider an inviscid and incompressible liquid jet of density $\rho$ and undisturbed radius $a$. The fluid is assumed to be a perfect conductor held at zero voltage and is concentrically placed inside a cylindrical electrode of radius $d>a$. The outer electrode is maintained at a constant voltage potential $V_{0}$ so that an electric field is set up in the dielectric annular region between the jet surface and the electrode-the annular region is assumed to be hydrodynamically passive and can be taken to be air, for example, with electric permittivity $\epsilon_{0}$. Considering axisymmetric deformations and utilizing cylindrical polar coordinates $(r, \theta, z)$, we denote the evolving jet surface by $r=S(z, t)$. The hydrodynamic problem in the region $0<r<S(z, t)$ is coupled with the electrostatic one in $S(z, t)<r<d$, through the normal stress balance that is modified by the electrical Maxwell stresses at the interface; the effect consequently appears in the Bernoulli equation as stated

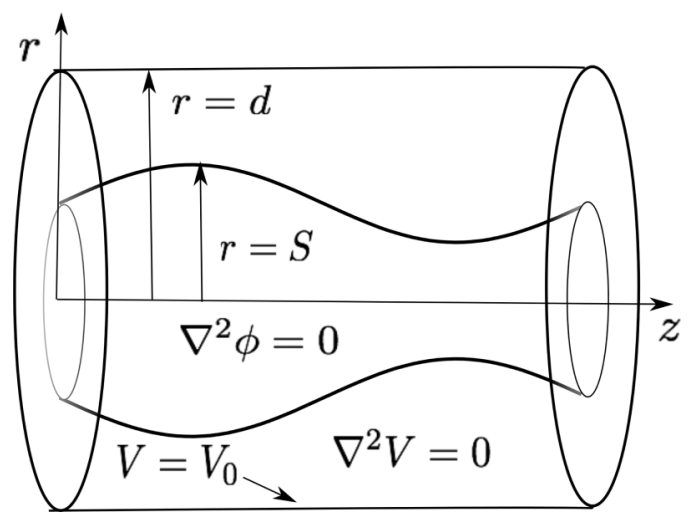

FIG. 1. Schematic of the problem showing the liquid thread placed concentrically inside a cylindrical electrode of radius $d$.

below; for details of such derivations, see Refs. [14,21,22], for example. A schematic of the problem is given in Fig. 1.

The flow is irrotational and so the velocity field is given by $\mathbf{u}=\nabla \phi$, where $\phi(r, z, t)$ is the fluid potential. Incompressibility then implies that $\phi$ is harmonic. The electric field is given by $\mathbf{E}=-\nabla V$, where $V(r, z, t)$ is the electrostatic potential, hence the field equations are

$$
\begin{aligned}
& \nabla^{2} \phi=0, \quad 0<r<S(z, t), \\
& \nabla^{2} V=0, \quad S(z, t)<r<d,
\end{aligned}
$$

the latter equation arising from Gauss's law $\operatorname{div}\left(\epsilon_{0} \mathbf{E}\right)=0$. At the jet axis $r=0$, we impose regularity of $\phi$ while at the interface $r=S(z, t)$ we need to satisfy a kinematic condition as well as the Bernoulli equation, namely

$$
\begin{gathered}
\phi_{r}=S_{t}+\phi_{z} S_{z}, \\
\rho \phi_{t}+\frac{1}{2} \rho\left(\phi_{r}^{2}+\phi_{z}^{2}\right)-\frac{\left(\epsilon_{0} / 2\right)}{\left(1+S_{z}^{2}\right)}\left(V_{r}-S_{z} V_{z}\right)^{2} \\
=-\frac{\gamma}{\left(1+S_{z}^{2}\right)^{3 / 2}}\left[\frac{1+S_{z}^{2}}{S}-S_{z z}\right]+K,
\end{gathered}
$$

where $K$ is a constant and $\gamma$ is the surface tension coefficient. Finally, the boundary conditions for the voltage potential $V$ are

$$
\begin{gathered}
V=0, \quad \text { on } \quad r=S(z, t), \\
V=V_{0}, \quad \text { on } \quad r=d .
\end{gathered}
$$

This completes the mathematical statement of the problem; we note that the hydrodynamic and electrostatic field Eqs. (1) and (2) are coupled through the Maxwell stresses appearing in the Bernoulli equation boundary condition, Eq. (4).

The following is an exact solution of the system Eqs. (1)-(6):

$$
\mathbf{u}=\mathbf{0}, \quad S(z, t)=a, \quad V=V_{0} \frac{\ln (r / a)}{\ln (d / a)} .
$$

This solution corresponds to a quiescent jet (note that any constant axial flow can be removed by a Galilean transformation) 
of uniform radius $a$, and with a perfectly radial electric field in the annular region $a<r<d$. In the absence of an electric field the perfectly cylindrical interface is susceptible to the Rayleigh-Plateau instability [2]—all linear disturbances with wavelengths longer than the unperturbed jet circumference are unstable, and sufficiently short waves are stable. Note that analogous results hold for viscous jets also, the main difference being in the magnitude of growth rates and their dependence on additional parameters; see the pioneering work of Rayleigh [23]. In the presence of a radial electric field, Huebner and Chu [24] have derived the modified dispersion relation for disturbances proportional to $\exp (i k z+\omega t)$ with $k$ the wave number and $\omega$ the growth rate, which in our notation reads (in dimensional variables)

$$
\begin{aligned}
& \left(\frac{\rho a^{3}}{\gamma}\right) \omega^{2} \\
& =\frac{k a I_{1}(k a)}{I_{0}(k a)}\left\{1-(k a)^{2}\right. \\
& \left.\quad-\frac{E_{b}}{\ln ^{2}(d / a)}\left[1+k a \frac{K_{0}(k d) I_{1}(k a)+I_{0}(k d) K_{1}(k a)}{I_{0}(k a) K_{0}(k d)-I_{0}(k d) K_{0}(k a)}\right]\right\},
\end{aligned}
$$

where

$$
E_{b}=\frac{\epsilon_{0} V_{0}^{2}}{\gamma a}
$$

is a dimensionless parameter measuring the strength of the electric field relative to capillary forces-it can be thought of as an electric Bond number. When $E_{b}=0$, the classical Rayleigh instability result follows; the presence of a field can stabilize long waves and this can be seen by considering Eq. (8) for small wave numbers $k a \ll 1$. The asymptotic result is

$$
\left(\frac{\rho a^{3}}{\gamma}\right) \omega^{2}=\frac{k^{2} a^{2}}{2}\left\{1-\frac{E_{b}[\ln (d / a)-1]}{\ln ^{3}(d / a)}\right\}+\ldots,
$$

and we observe that whenever $(d / a)>\mathrm{e} \simeq 2.7183$ and $E_{b}$ is sufficiently large, then $\omega^{2}<0$; i.e., linear waves are stable and dispersive. Physically, this requires the outer electrode to be sufficiently far from the undisturbed liquid surface and the applied electric field to be sufficiently strong-these findings are similar to problems where the liquid jet is highly viscous [21], or even with an annular fluid included [22]. For completeness in Fig. 2 we provide plots of the dispersion relation Eq. (8) as $E_{b}$ varies for a fixed value of $d / a=5$; the results clearly show the existence of long-wave instability in the absence of an electric field, and the emergence and enhancement of long-wave dispersive stabilization as $E_{b}$ is increased. Our aim in the remainder of this study is to describe the nonlinear dynamics in the presence of such dispersive effects and to derive Kortweg-de Vries-type equations that describe electrocapillary waves in cylindrical liquid threads.

\section{WEAKLY NONLINEAR THEORY AND DERIVATION OF KORTWEG-DE VRIES EQUATIONS}

In this section we construct nonlinear long wave solutions to the system Eqs. (1)-(6). In particular we assume that the typical axial wavelength of interfacial deformations, $\ell$ say,

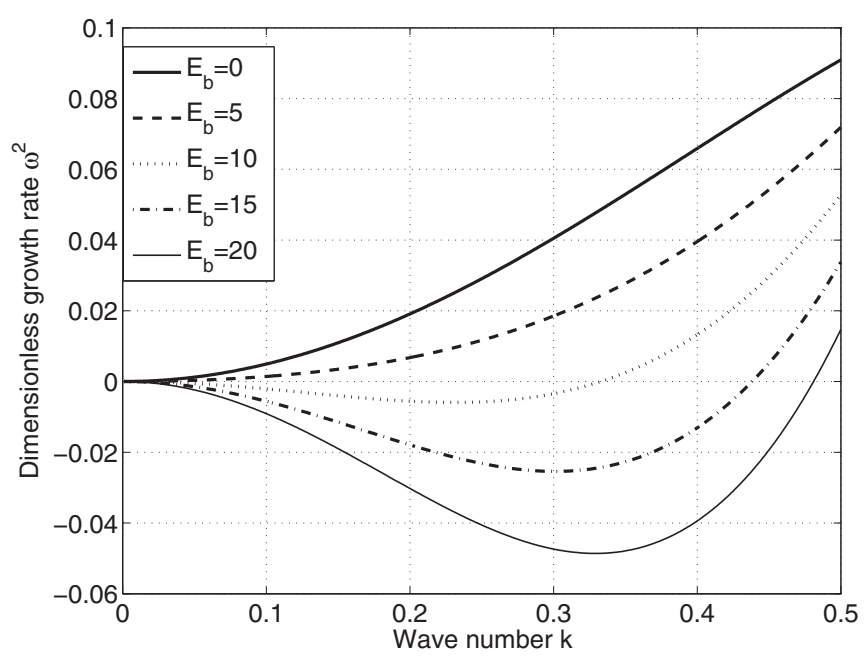

FIG. 2. Linear dispersion relation Eq. (8) showing stabilization of long waves due to the presence of an electric field; the outer electrode is at $d=5 a$. In the absence of a field, $E_{b}=0$, the classical Rayleigh instability is found (thick solid curve), while the waves become dispersively stable as $E_{b}$ increases from 5 to 20 as shown.

is long compared to the undisturbed jet radius $a$, so that the slenderness ratio

$$
\epsilon=\frac{a}{\ell} \ll 1
$$

We nondimensionalize the problem using the following scalings

$$
\begin{aligned}
& \phi=\left(\frac{\gamma \ell^{2}}{\rho a}\right)^{1 / 2} \phi^{\prime}, \quad V=V_{0} V^{\prime}, \quad r=a r^{\prime}, \quad z=\ell z^{\prime} \\
& S=a S^{\prime}, \quad t=\left(\frac{\rho a \ell^{2}}{\gamma}\right)^{1 / 2} t^{\prime} .
\end{aligned}
$$

Substituting Eq. (12) into Eqs. (1)-(6) and dropping the primes leads to the following equations and boundary conditions - the small parameter $\epsilon$ enters the problem and will be utilized in the development of asymptotic solutions later. (Note also that outer electrode radius $d$ becomes $D=d / a$ when nondimensionalized.)

$$
\begin{gathered}
\phi_{r r}+\frac{1}{r} \phi_{r}+\epsilon^{2} \phi_{z z}=0, \quad r=S(z, t), \\
V_{r r}+\frac{1}{r} V_{r}+\epsilon^{2} V_{z z}=0, \quad S(z, t)<r<D,
\end{gathered}
$$

The boundary conditions for the fluid potential at the interface $r=S$ are the kinematic and dynamic conditions

$$
\begin{gathered}
\phi_{r}=\epsilon^{2}\left(S_{t}+\phi_{z} S_{z}\right) \quad \text { on } \quad r=S, \\
\phi_{t}+\frac{1}{\left(1+\epsilon^{2} S_{z}^{2}\right)^{3 / 2}}\left[\frac{1+\epsilon^{2} S_{z}^{2}}{S}-\epsilon^{2} S_{z z}\right] \\
-\frac{1}{2} E_{b} \frac{1}{\left(1+\epsilon^{2} S_{z}^{2}\right)}\left(\frac{\partial V}{\partial r}-\epsilon^{2} S_{z} \frac{\partial V}{\partial z}\right)^{2} \\
+\frac{1}{2}\left[\frac{1}{\epsilon^{2}}\left(\frac{\partial \phi}{\partial r}\right)^{2}+\left(\frac{\partial \phi}{\partial z}\right)^{2}\right]=K \quad \text { on } \quad r=S(z, t),
\end{gathered}
$$


where $K$ is dimensionless analog of the constant appearing in Eq. (4), and the electric Bond number parameter $E_{b}$ has been defined in Eq. (9).

The boundary conditions for the voltage $V$ are

$$
\begin{aligned}
& V=0 \quad \text { on } \quad r=S, \\
& V=1 \quad \text { on } \quad r=D,
\end{aligned}
$$

where the former states that the jet interface is an equipotential since the fluid is a perfect conductor, while the second condition corresponds to the prescribed voltage at the outer wall.

The dimensionless system Eqs. (13)-(18) is exact but contains a small parameter $\epsilon$. Next we seek solutions for small $\epsilon$ with weakly nonlinear interfacial deformations, i.e., we write

$$
S(z, t)=1+\epsilon^{2} \eta(z, t)
$$

and assume the expansions

$$
\begin{gathered}
V(r, z, t)=V_{0}(r, z, t)+\epsilon^{2} V_{1}(r, z, t)+\epsilon^{4} V_{2}(r, z, t)+\ldots \\
\phi(r, z, t)=\epsilon^{2} \phi_{0}(r, z, t)+\epsilon^{4} \phi_{1}(r, z, t)+\ldots
\end{gathered}
$$

We also introduce the canonical Korteweg-de Vries scaling

$$
x=z-c t, \quad \tau=\epsilon^{2} t,
$$

where $c$ is to be determined-physically this means that we are looking for slowly evolving solutions on a timescale of order $1 / \epsilon^{2}$ in a frame of reference traveling with speed $c=\mathcal{O}(1)$. All the derivatives with respect to $z$ and $t$ in the basic equations are then rewritten in terms of derivatives with respect to $x$ and $\tau$ by using the transformations

$$
\frac{\partial}{\partial z}=\frac{\partial}{\partial x}, \quad \frac{\partial}{\partial t}=-c \frac{\partial}{\partial x}+\epsilon^{2} \frac{\partial}{\partial \tau} .
$$

Substituting Eqs. (19) and (20) into Eqs. (14), (17), and (18) gives at the lowest order

$$
V_{0 r r}+\frac{1}{r} V_{0 r}=0
$$

with the boundary conditions

$$
V_{0}=1 \quad \text { on } \quad r=D, \quad \text { and } \quad V_{0}=0 \quad \text { on } \quad r=1 .
$$

The solution of Eqs. (24) and (25) is

$$
V_{0} \equiv V_{0}(r)=\frac{\ln r}{\ln D} .
$$

At the order $\epsilon^{2}$ we have

$$
V_{1 r r}+\frac{1}{r} V_{1 r}=0
$$

subject to the boundary conditions

$$
\left.V_{1}\right|_{r=1}=-\eta \frac{1}{\ln D}, \quad \text { and }\left.\quad V_{1}\right|_{r=D}=0 .
$$

Note that the interfacial position depends on the slow timescale; i.e., $\eta \equiv \eta(x, \tau)$. The first of the condition Eqs. (28) follows from Eq. (17) after evaluating at the interfacial position given by Eq. (19) and expanding to order $\epsilon^{2}$. The solution of Eqs. (27) and (28) is

$$
V_{1}=\frac{\eta \ln r}{\ln ^{2} D}-\frac{\eta}{\ln D} .
$$

Proceeding to order $\epsilon^{4}$ we obtain the problem

$$
V_{2 r r}+\frac{1}{r} V_{2 r}=-V_{1 x x}
$$

subject to the boundary conditions

$$
\left.V_{2}\right|_{r=1}=-\left.V_{1 r}\right|_{r=1} \eta-\left.\frac{1}{2} V_{0 r r}\right|_{r=1} \eta^{2},
$$

and

$$
\left.V_{2}\right|_{r=D}=0 .
$$

Using the solution Eqs. (26) and (29) we can rewrite Eq. (31) as

$$
\left.V_{2}\right|_{r=1}=-\frac{\eta^{2}}{\ln ^{2} D}+\frac{\eta^{2} \ln D}{2} .
$$

The solution of Eq. (30) subject to the boundary condition Eqs. (32) and (33) is

$$
V_{2}=-\frac{\eta_{x x}}{\ln ^{2} D}\left(\frac{r^{2}}{4} \ln r-\frac{r^{2}}{4}\right)+\frac{r^{2} \eta_{x x}}{4 \ln D}+A \ln r+B
$$

where

$$
B=-\frac{\eta^{2}}{\ln ^{2} D}+\frac{\eta^{2}}{2 \ln D}-\frac{1}{4} \frac{\eta_{x x}}{\ln ^{2} D}-\frac{\eta_{x x}}{4 \ln D}
$$

and

$$
A=\frac{\eta_{x x}}{\ln ^{2} D}\left(\frac{D^{2}}{4}-\frac{1}{4} \frac{D^{2}}{\ln D}\right)-\frac{D^{2}}{4 \ln ^{2} D} \eta_{x x}-\frac{B}{\ln D} .
$$

For the fluid dynamics we substitute the expansion Eqs. (21) into the Laplace Eq. (13) and obtain the following solutions at the first three orders:

$$
\begin{gathered}
\phi_{0}=\phi_{0}(x, \tau), \\
\phi_{1}=-\frac{r^{2}}{4} \phi_{0 x x}+\theta_{1}(x, \tau), \\
\phi_{2}=\frac{r^{4}}{64} \phi_{0 x x x x}-\frac{r^{2}}{4} \theta_{1 x x}+\theta_{2}(x, \tau),
\end{gathered}
$$

where $\theta_{1}(x, \tau)$ and $\theta_{2}(x, \tau)$ are unknown functions. Note that all the terms that are singular at $r=0$ have been dropped from the solutions above. The boundary conditions to be imposed at $r=S$ are the kinematic and dynamic Eqs. (15) and (16), respectively. The former gives at the order $\epsilon^{4}$

$$
-\frac{1}{2} \phi_{0 x x}=-c \eta_{x}
$$

whereas the dynamic boundary condition Eq. (16) gives at order $\epsilon^{2}$

$$
-c \phi_{0 x}+\eta\left(E_{b} \frac{\ln D-1}{\ln ^{3} D}-1\right)=0 .
$$

Eliminating $\phi_{0}$ between Eqs. (38) and (39) by differentiating Eq. (39) with respect to $x$ yields

$$
-2 c^{2} \eta_{x}+\left(E_{b} \frac{\ln d-1}{\ln ^{3} d}-1\right) \eta_{x}=0
$$


which implies that

$$
c^{2}=\frac{1}{2} E_{b} \frac{\ln D-1}{\ln ^{3} D}-\frac{1}{2} .
$$

Equation (41) defines the velocity $c$ in terms of the basic variables. It requires $D>e$ and $E_{b}$ to be sufficiently large so that the right-hand side of Eq. (41) is positive. As expected, Eq. (41) is identical (after nondimensionalization) to the leading order long-wave dispersion relation given by Eq. (10).

In order to find equations for the unknown $\eta(x, \tau)$, we proceed to higher order. The kinematic boundary condition Eq. (15) gives at order $\epsilon^{6}$

$$
\eta_{\tau}+\phi_{0 x} \eta_{x}=\frac{1}{16} \phi_{0 x x x x}-\frac{1}{2} \theta_{1 x x}-\frac{1}{2} \phi_{0 x x} \eta,
$$

whereas the dynamic boundary condition Eq. (16) gives at the order $\epsilon^{4}$

$$
\begin{gathered}
\frac{c}{4} \phi_{0 x x x}-c \theta_{1 x}+\phi_{0 \tau}+\eta^{2}-\eta_{x x}-\frac{E_{b} \eta^{2}}{2 \ln ^{4} D}\left(3-5 \ln d+3 \ln ^{2} d\right) \\
-\frac{E_{b} \eta_{x x}}{4 \ln ^{4} d}\left(1-D^{2}+2 \ln D+2 \ln ^{2} D\right)+\frac{1}{2} \phi_{0 x}^{2}=0 .
\end{gathered}
$$

Next we eliminate $\theta_{1}$ between Eqs. (42) and (43) by first differentiating Eq. (43) with respect to $x$. This yields

$$
\begin{gathered}
2 c \eta_{\tau}+2 c \phi_{0 x} \eta_{x}+\frac{c}{8} \phi_{0 x x x x}+\phi_{0 x \tau}+\left(2-P_{1}\right) \eta \eta_{x} \\
-\left(1+P_{2}\right) \eta_{x x x}+\phi_{0 x} \phi_{0 x x}+c \phi_{0 x x} \eta=0,
\end{gathered}
$$

where $P_{1}$ and $P_{2}$ are defined as

$$
\begin{gathered}
P_{1}=\left[\frac{3 \ln ^{2} D-5 \ln D+3}{\ln ^{4} D}\right] E_{b}, \\
P_{2}=\left[\frac{2 \ln ^{2} D+2 \ln D+1-D^{2}}{4 \ln ^{4} D}\right] E_{b} .
\end{gathered}
$$

Relation Eqs. (38) and (39) imply that

$$
\phi_{0 x}=2 c \eta
$$

and on substituting this into Eq. (44) yields a single evolution equation for the jet shape $\eta(x, T)$ :

$$
\eta_{T}+\alpha \eta \eta_{x}+\beta \eta_{x x x}=0
$$

where

$$
\alpha=10 c^{2}+2-P_{1}, \quad \beta=\frac{c^{2}}{4}-P_{2}-1,
$$

and time has been rescaled according to $4 c \partial_{\tau} \rightarrow \partial_{T}$ (this is possible since $c>0$ ). The coefficients $\alpha$ and $\beta$ of the nonlinearity and dispersive terms, respectively, determine the type of soliton solutions that are supported. The classical solitons studied in water waves (see Whitham [25], for example) have $\alpha>0, \beta>0$ (e.g., $\alpha=1, \beta=6$ ) are functions of $\xi=x-s T$, where $s$ is the wave speed; solutions with $\alpha<0$ are also found in waves problems with an internal interface (see, for example, Dias and Vanden-Broeck [26], and references therein). Looking for such solutions in Eq. (48) and integrating twice with respect to $\xi$ yields [we also use the fact that $\eta(\xi) \rightarrow 0$ as $|\xi| \rightarrow \infty]$

$$
\beta\left(\frac{d \eta}{d \xi}\right)^{2}=s \eta^{2}-\frac{\alpha}{3} \eta^{3}
$$

If the amplitude of the wave is $\eta_{0}$ (this can be positive or negative), it follows by evaluating Eq. (50) at the crest and trough that the speed $s$ is given by

$$
s=\frac{1}{3} \alpha \eta_{0},
$$

and so Eq. (50) takes the form

$$
\beta\left(\frac{d \eta}{d \xi}\right)^{2}=\frac{\alpha}{3} \eta^{2}\left(\eta_{0}-\eta\right) .
$$

It is shown below that $\beta>0$ is a necessary condition for solitary waves to exist, and hence there are two possibilities: (i) $\alpha>0$ in which case $\eta<\eta_{0}$ with $\eta_{0}>0$, giving waves of elevation, and (ii) $\alpha<0$ in which case $\eta>\eta_{0}$ with $\eta_{0}<0$, giving waves of depression. From Eq. (51) for the speed we conclude that both elevation and depression waves have $s>0$ and hence their speed is supersonic relative to the linear speed $c$ [see Eq. (41)]. The solutions of Eq. (48) are the well-known solitary wave solutions

$$
u(x, T)=\frac{3 s}{\alpha} \operatorname{sech}^{2}\left[\frac{1}{2} \sqrt{\frac{s}{\beta}}(x-s T)\right],
$$

and as discussed above we obtain right-moving elevation or depression solitons for $\alpha>0$ or $\alpha<0$, respectively.

It remains to determine whether Eq. (48) with (49) can support both elevation and depression solitary waves. The parameters $\alpha$ and $\beta$ are functions of the dimensionless outer electrode radius $D$ and the electric-field parameter $E_{b}$ (large $E_{b}$ implies a large imposed electric field). Equation (41) for the speed imposes the constraint $\ln D>1$, and so we consider such values alone. In terms of $D$ and $E_{b}$ the expressions for $\alpha$ and $\beta$ are

$$
\begin{gathered}
\alpha=\frac{E_{b}}{\ln ^{4} D}\left[2 \ln ^{2} D-3\right]-3, \\
\beta=\frac{E_{b}}{8 \ln ^{4} D}\left[2 D^{2}-3 \ln ^{2} D-5 \ln D-2\right]-\frac{9}{8} .
\end{gathered}
$$

To determine regions in $D-E_{b}$ space where $\alpha$ and $\beta$ are positive or negative, it is useful to plot the curves $\alpha=0, \beta=0$. It can be seen from Eq. (54) that if $\ln D \leqslant \sqrt{\frac{3}{2}}$ then $\alpha<0$ for all positive values of $E_{b}$ ( $E_{b}<0$ is unphysical). The curves $\alpha=0, \beta=0$ are depicted in Fig. 3, and $\alpha, \beta$ are positive or negative above and below the curves, respectively, as indicated on the figure. The additional constraint $c^{2}>0$, with the speed given by Eq. (41), restricts permissible values of $D$ and $E_{b}$. The curve where $c^{2}=0$ is also included in Fig. 3 (solid curve), and we have $c^{2}>0$ above it. We can conclude, therefore, that for the waves found here we always have

$$
\beta>0 \text {, }
$$

while there are regions in parameter space where $\alpha$ can be positive or negative producing elevation or depression waves, respectively. In particular, $\alpha<0$ in the region that lies between the curves $c^{2}=0$ and $\alpha=0$, as indicated on Fig. 3. Typical waves are plotted in Fig. 4 for $\alpha>0$ (top panel) and $\alpha<0$ (bottom panel), respectively. These waves correspond to the pairs of parameter values $\ln D=1.17, E_{b}=12.5$ and $\ln D=$ $1.4, E_{b}=8$; these points are depicted by an open circle and a square, respectively, on Fig. 3. 


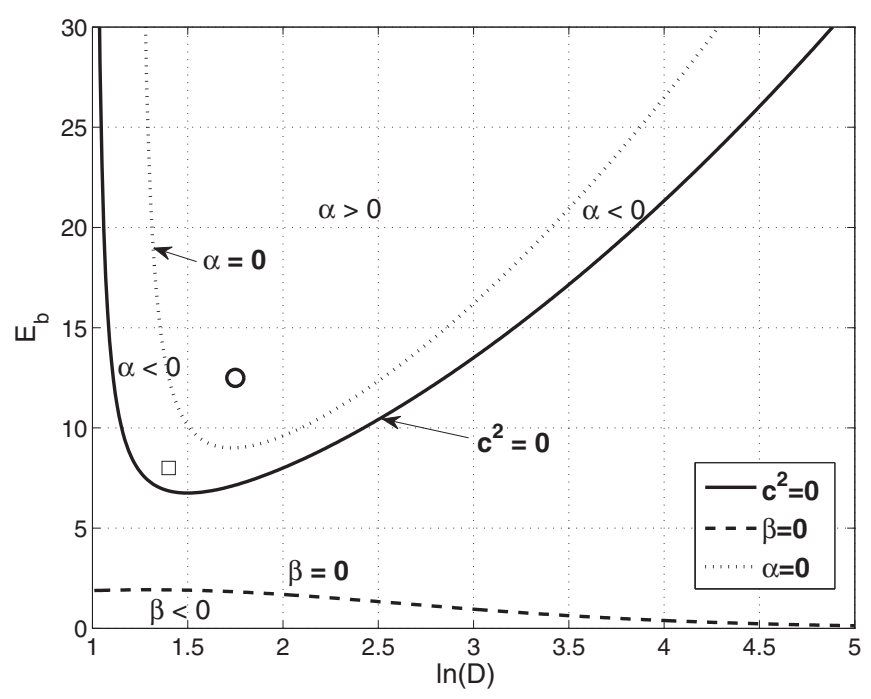

FIG. 3. Different regions in the $E_{b}-D$ plane where admissible solitary waves can be found. The curves indicate where $c^{2}=0$ (solid), $\beta=0$ (dashed), and $\alpha=0$ (dotted); the corresponding values of the parameters are positive above the curves and negative below the curves. The open circle indicates the point $\ln D=1.75, E_{b}=12.5$ [i.e., $\alpha=1.649, \beta=6.5874$ in Eq. (48)], and the square corresponds to $\ln D=1.4, E_{b}=8$ [i.e., $\alpha=-1.0841, \beta=3.5630$ in Eq. (48)]. The corresponding solitary waves are plotted in Fig. 4.

It is also worth noting that for values of $D$ and $E_{b}$ that give $\alpha=0$, the quadratic nonlinearity in the equation vanishes and a different asymptotic analysis is required to produce a higher order nonlinearity - the third derivative term cannot vanish as mentioned above-and so the system that derives is a Kortweg-de Vries equation with a high-order nonlinearity. This is not pursued further here and is left for future work.

It is useful to transform the scaled Kortweg-de Vries Eq. (48) back to original variables in order to demonstrate
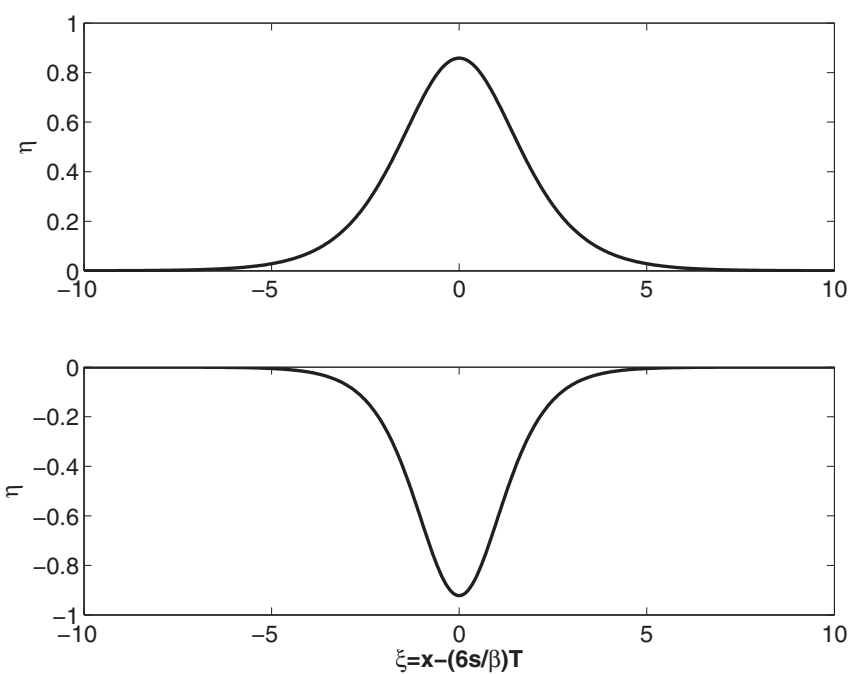

FIG. 4. Elevation (top) and depression (bottom) of solitary waves corresponding to the open circle and square, respectively, in Fig. 3; the parameter values are $\ln D=1.17, E_{b}=12.5$ and $\ln D=1.4$, $E_{b}=8$. the asymptotic balances of weak nonlinearity and weak dispersion. Recalling the nondimensionalization Eq. (12) and the asymptotic scalings $S=1+\epsilon^{2} \eta$ and time transformation $\partial_{t}=-c \partial_{x}+\epsilon^{2} \partial_{\tau}$ [see Eqs. (19) and (23)], the equation takes the following form in terms of dimensional variables:

$$
S_{t}+c^{*} S_{z}+\frac{\alpha^{*}}{4 c^{*}} \frac{(S-a)}{a} S_{z}+\frac{\beta^{*}}{c^{*}} a^{2} S_{z z z}=0,
$$

where $c^{*}=\left(\frac{\gamma}{\rho a}\right)^{1 / 2} c$ is the dimensional wave speed of linear long waves [the dimensional version of $c$ given by (41)], and $\alpha^{*}=\left(\frac{\gamma}{\rho a}\right) \alpha$ and $\beta^{*}=\left(\frac{\gamma}{\rho a}\right) \beta$ are dimensional parameters having units of velocity-squared and depending on the electric field and the geometric ratio $a / d$; see Eqs. (54) and (55). In the long-wave limit and for weakly nonlinear perturbations, Eq. (56) shows clearly the balances between nonlinearity and weak dispersion (after moving to a frame of reference of speed $c^{*}$ and introduction of a slow timescale as described in detail for the dimensionless equations in Sec. III).

\section{CONCLUSIONS}

We have considered the weakly nonlinear evolution of long-wave axisymmetric disturbances on a cylindrical liquid jet under the influence of a radially imposed electric field. We find that at sufficiently large imposed electric fields measured by the electric Bond number $E_{b}$ [see the definition just after Eq. (8)], and above a critical outer electrode radius $D=d / a>e \approx 2.7183$, long waves are dispersive and an asymptotic analysis analogous to that used to derive the Korweg-de Vries equation for water waves (see Whitham [25]) is applicable and leads to the KdV Eq. (48). The coefficients $\alpha$ and $\beta$ of the nonlinear and dispersive terms, respectively, depend on the two parameters $E_{b}$ and $D$, and admissible values are additionally constrained by the condition $c^{2}>0$, where the latter is given by Eq. (41). It is found that for the theory to hold we must have $\beta>0$, whereas $\alpha$ can be positive or negative as indicated in the phase diagram in Fig. 3. If $\alpha>0$ we obtain right-moving solitary waves of elevation, while for $\alpha<0$ depression solitons emerge; see Fig. 4 for representative solutions. We note that similar waves were found in the case of ferrofluids (see the Introduction), but interestingly the mathematical problems are quite different; in the present problem a Laplace equation for the electric field must be solved in the annulus in order to find the appropriate term in the Bernoulli equation, whereas for the ferrofluid problem there is a decoupling and the Bernoulli equation is simply modified by a term inversely proportional to the local jet radius. We also note that the solutions constructed here are likely to be susceptible to shorter wave disturbances that would be modulated by the soliton envelope-such calculations are beyond the scope of the present work and would most likely require time-dependent direct numerical simulations. This is left for future work.

\section{ACKNOWLEDGMENTS}

The work of D.T.P. and J.-M.V.B. was partly supported by the Engineering and Physical Sciences Research Council of Great Britain by Grants No. EP/K041134/1 and No. EP/J019569/1. 
[1] J. Plateau, Statique experimentale et theorique des liquides soumis aux seules forces moleculaires, Acad. Sci. Bruxelles Mem. 23, 5 (1849).

[2] Lord Rayleigh, On the instability of jets, Proc. Lond. Math. Soc. 10, 4 (1878).

[3] R. J. Raco, Electrically supported column of liquid, Science 160, 311 (1968).

[4] A. Ramos, H. González, and A. Castellanos, Experiments on dielectric liquid bridges subjected to axial electric fields, Phys. Fluids 6, 3206 (1994).

[5] C. L. Burcham and D. A. Saville, The electrohydrodynamic stability of a liquid bridge: Microgravity experiments on a bridge suspended in a dielectric gas, J. Fluid Mech. 405, 37 (2000).

[6] D. Volkov, D. T. Papageorgiou, and P. G. Petropoulos, Accurate and efficient boundary integral methods for electrified liquid bridge problems, SIAM J. Sci. Comput. 26, 2102 (2005).

[7] A. B. Basset, Waves and jets in a viscous liquid, Am. J. Math. 16, 93 (1894).

[8] J. M. Schneider, N. R. Lindbald, C. E. Hendricks, and J. M. Crowley, Stability of an electrified jet, J. Appl. Phys. 38, 2599 (1967).

[9] J. Neukermans, Stability criteria of an electrified liquid jet, J. Appl. Phys. 44, 4769 (1973).

[10] G. Artana, H. Romat, and G. Touchard, Theoretical analysis of linear stability of electrified jets flowing at high velocity inside a coaxial electrode, J. Electrost. 43, 83 (1998).

[11] G. Artana, G. Touchard, and H. Romat, Absolute and convective instabilities in an electrified jet, J. Electrost. 40-41, 33 (1997).

[12] A. Chauhan, C. Maldarelli, D. T. Papageorgiou, and D. S. Rumschitzki, The absolute instability of an inviscid compound jet, J. Fluid Mech. 549, 81 (2006).

[13] E. R. Setiawan and S. D. Heister, Nonlinear modeling of an infinite electrified jet, J. Electrost. 42, 243 (1997).
[14] S. Grandison, J.-M. Vanden-Broeck, D. T. Papageorgiou, T. Miloh, and B. Spivak, Axisymmetric waves in electrohydrodynamic flows, J. Eng. Math. 62, 133 (2007).

[15] A. P. Bassom, M. G. Blyth, and D. T. Papageorgiou, Using surfactants to stabilize two-phase pipe flows of core-annular type, J. Fluid Mech. 704, 333 (2012).

[16] J.-M. Vanden-Broeck, T. Miloh, and B. Spivak, Axisymmetric capillary waves, Wave Motion 27, 245 (1998).

[17] V. Bashtovoi, A. Rex, and R. Foigel, Some nonlinear wave processes in a magnetic fluid, J. Magn. Magn. Mater. 39, 115 (1983).

[18] D. Rannacher and A. Engel, Cylindrical Korteweg-de Vries solitons on a ferrofluid surface, New J. Phys. 8, 108 (2006).

[19] E. Bourdin, J.-C. Bacri, and E. Falcon, Observation of axisymmetric solitary waves on the surface of a ferrofluid, Phys. Rev. Lett. 104, 094502 (2010).

[20] M. G. Blyth and E. Parau, Solitary waves on a ferrofluid jet, J. Fluid Mech. 750, 401 (2014).

[21] Q. Wang, S. Mählmann, and D. T. Papageorgiou, Dynamics of liquid jets and threads under the action of radial electric fields: Microthread formation and touchdown singularities, Phys. Fluids 21, 032109 (2009).

[22] Q. Wang and D. T. Papageorgiou, Dynamics of a viscous thread surrounded by another viscous fluid in a cylindrical tube under the action of a radial electric field: Breakup and touchdown singularities, J. Fluid Mech. 683, 27 (2011).

[23] Lord Rayleigh, On the instability of a cylinder of viscous liquid under the capillary force, Philos. Mag. 34, 145 (1892).

[24] A. L. Huebner and H. N. Chu, Instability and breakup of charged liquid jets, J. Fluid Mech. 49, 361 (1971).

[25] G. B. Whitham, Linear and Nonlinear Waves (WileyInterscience, New York, 1974).

[26] F. Dias and J.-M. Vanden-Broeck, On internal fronts, J. Fluid Mech. 479, 145 (2003). 